\title{
Surgery for IDH1/2 wild-type glioma invading the corpus callosum
}

\author{
Pamela Franco ${ }^{1,2}$ (D) Daniel Delev ${ }^{3} \cdot$ Debora Cipriani $^{1,2} \cdot$ Nicolas Neidert $^{1,2} \cdot$ Elias Kellner $^{2,4} \cdot$ Waseem Masalha ${ }^{1,2}$. \\ Bianca Mercas ${ }^{1,2} \cdot$ Irina Mader $^{2,5} \cdot$ Peter Reinacher ${ }^{2,6} \cdot$ Astrid Weyerbrock $^{2} \cdot$ Christian Fung $^{1,2} \cdot$ Jürgen Beck ${ }^{1,2}$. \\ Dieter Henrik Heiland ${ }^{1,2}$. Oliver Schnell ${ }^{1,2}$
}

Received: 3 August 2020 / Accepted: 16 October 2020 / Published online: 23 October 2020

(C) The Author(s) 2020

\begin{abstract}
Background Glioblastoma of the corpus callosum (ccGBM) are rare tumors, with a dismal prognosis marked by a rapid clinical deterioration. For a long time, surgical treatment was not considered beneficial for most patients with such tumors. Recent studies claimed an improved survival for patients undergoing extensive resection, albeit without integration of the molecular profile of the lesions. The purpose of this study was to investigate the effect of biopsy and surgical resection on oncological and functional outcomes in patients with IDH wild-type ccGBM.

Methods We performed a retrospective analysis of our institution's database of patients having been treated for high-grade glioma between 2005 and 2017. Inclusion criteria were defined as follows: patients older than 18 years, histopathological, and molecularly defined IDH wild-type glioma, major tumor mass (at least 2/3) invading the corpus callosum in the sagittal plane with a uni- or bilateral infiltration of the adjacent lobules. Surgical therapy (resection vs. biopsy), extent of resection according to the remaining tumor volume and adjuvant treatment as well as overall survival and functional outcome using the Karnofsky Performance Score (KPS) were analyzed.

Results Fifty-five patients were included in the study, from which the mean age was 64 years and men $(n=34,61.8 \%)$ were more often affected than women $(n=21,38.2 \%)$. Thirty $(54.5 \%)$ patients were treated with stereotactic biopsy alone, while 25 patients received tumor resection resulting in $14.5 \%(n=8)$ gross-total resections and $30.9 \%(n=17)$ partial resections. The 2 -year survival rate after resection was $30 \%$ compared to $7 \%$ after biopsy $(p=0.047)$. The major benefit was achieved in the group with gross-total resection, while partial resection failed to improve survival. Neurological outcome measured by KPS did not differ between both groups either pre- or postoperatively.

Conclusions Our study suggests that in patients with corpus callosum glioblastoma, gross-total resection prolongs survival without negatively impacting neurological outcome as compared to biopsy.
\end{abstract}

This article is part of the Topical Collection on Tumor - Glioma

Previous presentation Portions of this study were presented in oral presentation form at the 68th Annual Meeting of the German Society of Neurosurgery (DGNC) in Magdeburg on May 15, 2017.

Electronic supplementary material The online version of this article (https://doi.org/10.1007/s00701-020-04623-z) contains supplementary material, which is available to authorized users.

Pamela Franco

pamela.heiland@uniklinik-freiburg.de

1 Department of Neurosurgery, Medical Center - University of Freiburg, Breisacher Straße 64, 79106 Freiburg, BW, Germany

2 Faculty of Medicine, University of Freiburg, Freiburg, BW, Germany

3 Department of Neurosurgery, University of Aachen, Aachen, NRW, Germany
4 Department of Radiology, Medical Centre - University of Freiburg, Freiburg, BW, Germany

5 Specialist Centre for Radiology, Schoen Clinic, Vogtareuth, BY, Germany

6 Department of Neurosurgery, Division Stereotactic and Functional Neurosurgery, Medical Center- University of Freiburg, Freiburg, BW, Germany 
Keywords Corpus callosum glioblastoma $\cdot$ Butterfly glioblastoma $\cdot$ Glioblastoma $\cdot$ Neurosurgery $\cdot$ IDH status $\cdot$ Molecular diagnosis $\cdot$ Extent of resection

\begin{tabular}{ll}
\multicolumn{2}{l}{ Abbreviations } \\
ccGBM & Glioblastoma of the corpus callosum \\
IDH & Isocitrate dehydrogenase \\
CNS & Central nervous system \\
KPS & Karnofsky Performance Score \\
OS & Overall survival \\
MR & Magnetic resonance \\
RANO & Response Assessment in Neuro-Oncology criteria
\end{tabular}

\section{Introduction}

Glioblastomas are the most common malignant astroglialderived tumors accounting for $47.1 \%$ of all central nervous system (CNS) tumors with an incidence of 3.20 per 100,000 persons per year [1]. Despite best available treatment, the median overall survival rate is about 16 months [2-5]. In general, glioblastoma treatment is based on attempting complete resection of contrast-enhancing tumor followed by adjuvant therapy [6]. However, treatment for glioblastomas initially infiltrating the corpus callosum remains controversial [7-9]. These tumors present with a characteristic growth pattern that invades more or less both cerebral hemispheres. Most commonly, they arise within the frontal lobe but can also be found in the parietal and occipital lobes. If both hemispheres are almost equally infiltrated, they are referred to as butterfly glioblastomas $[8,10,11]$. Clinically, these tumors have a wide range of symptomatic presentations including disorientation, focal epilepsy as well as comatose, and mute states $[8,11]$. Since these tumors invade eloquent areas, complete resection is often unlikely. Furthermore, several authors argue that even the attempt of gross-total resection would be too aggressive and may lead to a more rapid clinical deterioration and delay adjuvant treatment $[8,9]$. Therefore, until lately, diagnostic and molecular profiling via tumor biopsy was widely favored over the attempt of gross-total tumor resection. Yet, a growing number of authors have published data highlighting the benefits of resection of glioblastoma of the corpus callosum (ccGBM), specifically regarding improved overall survival without aggravating persistent neurological deficits [12-14]. Unfortunately, there is a lack of consistent molecular profiling of the tumors in the previous studies, which leads to a disputable interpretation of the benefits of surgical resection upon survival in this subset of tumors. An IDH mutation is an independent prognostic factor that improves overall survival (OS) in glioblastoma [15]. The aim of this study was to explore the impact of tumor resection and adjuvant treatment strategies on IDH wild-type corpus callosum glioblastoma.

\section{Materials and methods}

\section{Patient data acquisition}

We screened all patients treated for malignant brain tumors at the Department of Neurosurgery at the Medical CenterUniversity of Freiburg between 2005 and 2017. Eligibility criteria were a suspicion of corpus callosum glioblastoma on MR imaging, later confirmed by histopathology and molecular profiling, on adult patients (older than 18 years of age). Patients with multilocular glioblastoma (i.e., infiltration of more than three lobes) as well as patients with glioblastoma manifestation distant from the corpus callosum were excluded from the study. Patient demographics are listed in Table 1. Clinical data were extracted from medical archives. Written informed consent for utilization of clinical data for scientific purposes was obtained preoperatively from all patients. The local ethics committee of the University of Freiburg approved data evaluation (protocol 472/15).

\section{Histopathological and molecular diagnostics}

Specimens were fixed in $4 \%$ phosphate-buffered formaldehyde and paraffin-embedded according to standard procedures in the Institute of Neuropathology, Medical CenterUniversity of Freiburg, as detailed described in our previous works $[16,17]$. In order to determine the IDH mutation status, we performed IHC (R132H). In patients younger than 65 years, we used next-generation sequencing of IDH1 and IDH2 to confirm negative staining results.

\section{MR imaging acquisition}

Imaging was performed on 1.5-T and 3-T whole body system (Siemens Magnetom Avanto, Trio, or Prisma, Erlangen, Germany). Anatomical imaging consisted of 3D T1weighted sequences (MPRAGE, TR $2300 \mathrm{~ms}$, TI $988 \mathrm{~ms}$, TE $2.26 \mathrm{~ms}$, voxel size $1 \mathrm{~mm}^{3}$ ) before and after contrast application $(0.1 \mathrm{mM}$ gadoteridol per $\mathrm{kg}$ body weight (ProHance ${ }^{\circledR}$, Bracco, Konstanz, Germany)) and 2D T2weighted turbo spin-echo sequence (TR $4500 \mathrm{~ms}$, TE $100 \mathrm{~ms}$, echo train length 17 , voxel size $0.6 \times 0.6 \times 2 \mathrm{~mm}^{3}$ ). Patients received a postoperative MRI scan with and without contrast enhancement within the first $72 \mathrm{~h}$ after surgical resection and regular MRI scans every 3 months from then on. Tumor progression was defined according to RANO-criteria [18]. Extent of resection was assessed on pre- and postoperative MRI; gross-total resection was defined when less than 5\% remaining tumor was detected [19]. 
Table 1 Patient demographics

\begin{tabular}{|c|c|c|c|c|c|c|c|}
\hline & \multicolumn{2}{|c|}{ Entire cohort } & \multicolumn{2}{|c|}{ Surgical resection } & \multicolumn{3}{|c|}{ Biopsy } \\
\hline & $N$ & $\%$ & $N$ & $\%$ & $N$ & $\%$ & $p$ \\
\hline Patients & 55 & 100 & 25 & 45.5 & 30 & 54.5 & - \\
\hline \multicolumn{8}{|l|}{ Gender } \\
\hline Female & 21 & 38.2 & 10 & 40 & 11 & 36.7 & 0.82 \\
\hline Male & 34 & 61.8 & 15 & 60 & 19 & 63.3 & 0.82 \\
\hline \multicolumn{8}{|l|}{ Age in years } \\
\hline Median (IQR) & 66 & $(57.5-73)$ & 62.3 & $(50.5-66.9)$ & 68.5 & $(62.1-76)$ & - \\
\hline \multicolumn{8}{|l|}{ Symptomatic at first presentation } \\
\hline Memory impairment & 15 & 27.3 & 3 & 12 & 12 & 40 & 0.02 \\
\hline Confusion/mental status alteration & 38 & 69 & 17 & 68 & 21 & 70 & 0.87 \\
\hline Visual field deficit & 6 & 11 & 4 & 16 & 2 & 6.7 & 0.27 \\
\hline Aphasia & 10 & 18.2 & 7 & 28 & 3 & 10 & 0.08 \\
\hline Cranial nerve impairment & 2 & 3.6 & 1 & 4 & 1 & 3.3 & 0.89 \\
\hline Motor function impairment & 13 & 23.6 & 7 & 28 & 6 & 20 & 0.49 \\
\hline Sensory function impairment & 3 & 5.5 & 1 & 4 & 2 & 6.7 & 0.66 \\
\hline Psychiatric alteration & 1 & 1.8 & 0 & - & 1 & 3.3 & 0.36 \\
\hline Headache & 7 & 12.7 & 6 & 24 & 1 & 3.3 & 0.01 \\
\hline Epilepsy & 11 & 20 & 3 & 12 & 8 & 26.7 & 0.20 \\
\hline
\end{tabular}

$I Q R$, interquantile range

\section{Tumor segmentation}

From the patients with complete and qualitatively acceptable 3D MR imaging $(n=36)$, tumor segmentation was performed. First, images were converted to a NifTI format and loaded into a Web-based framework for medical imaging analysis (http:// www.nora-imaging.com). Segmentation was then carried out by a semi-automated approach and processed in R-software. Tumor volumetry was performed based on the contrastenriched tumor region.

\section{Surgical treatment}

Groups were divided based on the main treatment strategy (gross-total or partial resection and stereotactic biopsy). Patients who underwent surgical resection within the first month after stereotactic biopsy were assigned to the resection group.

\section{Oncological treatment}

Patients received glioblastoma-specific oncological treatment according to the protocol defined by Stupp et al. in 2005 whenever patients consented to the treatment and if they had a good clinical status (Karnofsky Performance Scale (KPS) > $70 \%$ ). Alternative treatments, i.e., either chemotherapy with temozolomide or lomustine alone or radiotherapy alone, were also present in the study.

\section{Primary endpoint}

The primary endpoint was overall survival after first diagnosis, which was defined as the time until death from any cause (patients with event) or until last contact (patients without event). Secondary endpoints were surgical and neurological outcomes. For the latter, we used the KPS and clinical parameters.

\section{Statistical analysis}

We used the Kaplan-Meier method to estimate survival distributions and a Cox proportional-hazards model to calculate hazard ratios. Statistical power was measured by "powerSurvEpi," an R-software package. With a calculated power over $80 \%$, we set the alpha level to $5 \%(p<0.05)$ for statistical significance. Patients lost at follow-up where censored at the recorded date of last contact. Numeric variables were tested for normal distribution by ShapiroWilks test. In case of a normal distribution, variables were represented by mean and standard deviation and tested by non-paired Student's $t$ test. In case of non-normal distribution, variables were presented with median and interquantile range (IQR), tested by Wilcoxon rank sum test. Nominal variables were tested by either chi-square or Fisher's exact test. 


\section{Results}

\section{Patients' demographics}

A total of 2720 patients were treated for high-grade glioma between 2005 and 2017 at the Medical Center-University of Freiburg. Fifty-five adult patients with molecularly defined IDH wild-type glioma of the corpus callosum were included in the study. Median age was 66 years (IQR 57.5-73). Male patients accounted for $61.8 \%(n=34)$ of the cases, while females represented $38.2 \%(n=21)$. The leading clinical symptom at first presentation was confusion/alteration of mental status in $69 \%$ of patients followed by memory impairment (27.3\%), motor function impairment (23.6\%), and epilepsy (20\%). Other clinical symptoms included aphasia (18.2\%), headache $(12.7 \%)$, and visual field deficit (11\%) and less common were sensory function impairment $(5.5 \%)$, cranial nerve impairment (3.6\%), and psychiatric alteration (1.8\%). Regarding the anatomical invasion of the corpus callosum, the genu was significantly more often invaded $(p=0.005)$.
Characteristics of the biopsy and resection cohorts

Thirty patients (54.6\%) received a biopsy to confirm the histology from which $50 \%$ were further treated by chemotherapy alone $(n=6,20 \%)$ or combined radiotherapy plus chemotherapy $(n=9,30 \%)$. Twenty-five patients were treated by surgical resection from which 8 patients received a gross-total resection, and 17 patients were partially resected. The decision towards resection over biopsy was mainly driven by high tumor burden and mass effect of the tumor which was confirmed by our volumetric evaluation. The mean volume in the group of resected patients was significantly greater compared the biopsy group (resection: $59.4 \mathrm{~cm}^{3}$, biopsy $35.2 \mathrm{~cm}^{3}, p=$ 0.01 ). Postoperative volume after resection was decreased by tenfold (mean volume: $5.96 \mathrm{~cm}^{3}$ ). The mean postoperative tumor volume in the biopsy group remained constant. Received adjuvant treatment was well balanced in both groups regarding treatment modalities and duration of the treatment. In our cohort, we found that gliomas localized in the genu were preferentially resected in comparison to other anatomical

\begin{tabular}{|c|c|c|c|c|c|c|c|}
\hline & \multicolumn{2}{|c|}{ Entire cohort } & \multicolumn{2}{|c|}{ Surgical resection } & \multicolumn{3}{|c|}{ Biopsy } \\
\hline & $N$ & $\%$ & $N$ & $\%$ & $N$ & $\%$ & $p$ \\
\hline Patients & 55 & 100 & 25 & 45.5 & 30 & 54.5 & - \\
\hline \multicolumn{8}{|l|}{ Site of CC invasion } \\
\hline Rostrum & 1 & 1.8 & 0 & 0 & 1 & 3.3 & 0.36 \\
\hline Genu & 26 & 47.2 & 17 & 68 & 9 & 30 & 0.005 \\
\hline Genu/body & 5 & 9 & 2 & 8 & 3 & 10 & 0.79 \\
\hline Body & 11 & 20 & 3 & 12 & 8 & 26.7 & 0.18 \\
\hline Body/splenium & 6 & 11 & 1 & 4 & 5 & 16.7 & 0.13 \\
\hline Splenium & 6 & 11 & 2 & 8 & 4 & 13.3 & 0.53 \\
\hline \multicolumn{8}{|l|}{ Surgery } \\
\hline GTR & 8 & 14.55 & & & & & \\
\hline PR & 17 & 30.9 & & & & & \\
\hline Biopsy & 30 & 54.55 & & & & & \\
\hline \multicolumn{8}{|l|}{ Tumor volume in $\mathrm{cm}^{3}$} \\
\hline Preoperative median & 43.5 & & 59.4 & & 35.2 & & 0.01 \\
\hline Postoperative median & 5.96 & & 5.96 & & 35.2 & & $*$ \\
\hline \multicolumn{8}{|l|}{ Complication } \\
\hline None & 42 & 76.3 & 17 & 68 & 25 & 83.3 & 0.18 \\
\hline Hemorrhage & 3 & 5.4 & 3 & 12 & 0 & 0 & 0.05 \\
\hline Ischemia & 1 & 1.8 & 1 & 4 & 0 & 0 & 0.27 \\
\hline Cranial nerve deficit & 1 & 1.8 & 1 & 4 & 0 & 0 & 0.27 \\
\hline Meningitis & 2 & 3.6 & 2 & 8 & 0 & 0 & 0.84 \\
\hline Hydrocephalus & 3 & 5.4 & 1 & 4 & 2 & 6.7 & 0.81 \\
\hline Aphasia & 3 & 5.4 & 1 & 4 & 2 & 6.7 & $<0.001$ \\
\hline Pneumonia & 1 & 1.8 & 0 & 0 & 1 & 3.3 & 0.89 \\
\hline
\end{tabular}

$I Q R$, interquantile range; $C C$, corpus callosum; GTR, gross-total resection; $P R$, partial resection 
locations (resection $n=17$ vs. biopsy $n=9, p=0.005$ ), Table 2.

\section{Follow-up outcome}

Regarding functional outcome, we found that patients in both the surgery and biopsy groups had an improvement of their KPS immediately postoperative, and in the first 2 weeks after the hospital stay, this is most probably due to the effect of steroid treatment perioperatively. The surgery group contained $52.9 \%$ of all patients with a KPS below $80 \%$ before hospitalization, which improved to only $33.3 \%$ patients with a KPS below $80 \%$ after hospitalization. In the biopsy group, patients with a KPS under $80 \%$ also saw a similar reduction from 69.6 to $33.3 \%$ after hospital stay (Table 3). A significant difference between both surgery and biopsy was not detected. At the 3-and 12-month follow-up, the number of patients with a KPS below $80 \%$ was constantly increasing in the biopsy group (KPS < 80\% at 3 months: $82 \%$, at 12 months: 92\%) and the partial resection group (KPS $<80 \%$ at 3 months: $86.6 \%$, at 12 months: $86.6 \%$ ). The only significant difference was found in the gross-total resection group, where $60 \%$ of the patients maintained a KPS over $80 \%$ at 3-month follow-up $(p=0.027)$. At 12 -month follow-up, only 5 patients presented with a KPS over $80 \%$; here, no significant difference between biopsy or surgery was detected (Table 4).

\section{Surgical complications}

Thirteen patients (23.6\%) had postoperative complications. In the surgical resection group, the leading complications were postoperative hemorrhage in the resection cavity $(n=3)$ and meningitis $(n=2)$. Other complications included territorial

Table 3 Oncology characteristics

\begin{tabular}{|c|c|c|c|c|c|c|c|}
\hline & \multicolumn{2}{|c|}{ Entire cohort } & \multicolumn{2}{|c|}{ Surgical resection } & \multicolumn{3}{|c|}{ Biopsy } \\
\hline & $N$ & $\%$ & $N$ & $\%$ & $N$ & $\%$ & $p$ \\
\hline Patients & 55 & 100 & 25 & 45.5 & 30 & 54.5 & - \\
\hline \multicolumn{8}{|c|}{ Adjuvant treatment } \\
\hline $\mathrm{RCT}$ & 16 & 29.1 & 7 & 28 & 9 & 30 & 0.87 \\
\hline $\mathrm{CT}$ & 13 & 23.4 & 7 & 28 & 6 & 20 & 0.49 \\
\hline $\mathrm{RT}$ & 3 & 5.5 & 3 & 12 & 0 & 0 & 0.05 \\
\hline Palliative & 23 & 41.8 & 8 & 32 & 15 & 50 & - \\
\hline \multicolumn{8}{|c|}{ MGMT-promoter status } \\
\hline Methylated & 15 & 27.3 & 4 & 16 & 11 & 36.6 & 0.09 \\
\hline Unmethylated & 15 & 27.3 & 9 & 36 & 6 & 20 & 0.18 \\
\hline NA & 25 & 45.4 & 12 & 48 & 13 & 43.4 & - \\
\hline
\end{tabular}

$R C T$, radiochemotherapy; $C T$, chemotherapy; $R T$, radiotherapy; $N A$, not available
Table 4 Karnofsky Performance Score

\begin{tabular}{|c|c|c|c|c|c|c|c|}
\hline & \multicolumn{2}{|c|}{ Entire cohort } & \multicolumn{2}{|c|}{ Surgical Resection } & \multicolumn{3}{|c|}{ Biopsy } \\
\hline & $N$ & $\%$ & $N$ & $\%$ & $N$ & $\%$ & $p$ \\
\hline Patients & 55 & 100 & 25 & 45.5 & 30 & 54.5 & - \\
\hline \multicolumn{8}{|c|}{ Preoperative } \\
\hline 100 & 0 & - & 0 & - & 0 & - & - \\
\hline 90 & 17 & 30.9 & 10 & 40 & 7 & 23.3 & 0.18 \\
\hline 80 & 23 & 41.8 & 8 & 32 & 15 & 50 & 0.18 \\
\hline$<80$ & 15 & 27.3 & 7 & 28 & 8 & 26.7 & 0.91 \\
\hline \multicolumn{8}{|c|}{ Postoperative } \\
\hline 100 & 13 & 23.6 & 9 & 36 & 4 & 13.3 & 0.05 \\
\hline 90 & 22 & 40 & 8 & 32 & 14 & 46.7 & 0.27 \\
\hline 80 & 6 & 10.9 & 4 & 16 & 2 & 6.7 & 0.27 \\
\hline$<80$ & 14 & 25.5 & 4 & 16 & 10 & 33.3 & 0.14 \\
\hline \multicolumn{8}{|c|}{ 3-month follow-up } \\
\hline 100 & 5 & 9.1 & 4 & 16 & 1 & 3.33 & 0.10 \\
\hline 90 & 7 & 12.7 & 3 & 12 & 4 & 13.33 & 0.88 \\
\hline 80 & 6 & 10.9 & 2 & 8 & 4 & 13.33 & 0.53 \\
\hline$<80$ & 12 & 21.8 & 7 & 28 & 5 & 16.7 & 0.31 \\
\hline 0 & 25 & 45.5 & - & - & - & - & - \\
\hline \multicolumn{8}{|c|}{ 12-month follow-up } \\
\hline 100 & 4 & 7.3 & 2 & 8 & 2 & 6.7 & 0.85 \\
\hline 90 & 1 & 1.8 & 1 & 4 & 0 & - & 0.27 \\
\hline 80 & 0 & - & - & - & - & - & - \\
\hline$<80$ & 10 & 18.2 & 7 & 28 & 3 & 10 & 0.08 \\
\hline 0 & 40 & 72.7 & - & - & - & - & - \\
\hline
\end{tabular}

infarction leading to decompressive hemicraniectomy, cranial nerve deficit, hydrocephalus requiring emergency external ventricle drainage placement, and aphasia ( $n=1$ each). In the biopsy group, 2 patients developed postoperative hydrocephalus requiring emergency external ventricle drainage placement, 2 patients developed aphasia, and one patient had an acquired bacterial pneumonia requiring intensive care. All other clinical features remained well balanced between both groups; clinical characteristics are listed in Table 1 and surgical characteristics are listed in Table 2.

\section{Outcome analysis of biopsy vs. resection}

We analyzed the effect of a surgical resection on overall survival (primary outcome). Forty-five patients reached the defined endpoint (resection $n=18$, biopsy $n=27$ ), while 10 patients were censored (resection $n=7$, biopsy $n=3$ ). We used a Cox proportional-hazards model to estimate the outcome differences, which resulted in a significantly improved survival for patients who underwent a resection (258 days CI 95\% 153-413 days) compared to those biopsied (220 days CI $95 \%$ NA), Fig. 1. The difference of the median survival was 
found to be relatively small, but the 2-year survival rate was increased from $7 \%$ of biopsy patients to $30 \%$ in the resection group ( $p=0.047$ ). In order to confirm our findings, we used a multivariate approach by Cox proportional-hazards model including other outcome relevant parameters such as the presence of epilepsy, the anatomical location of the tumor, the type of adjuvant treatment, age, and KPS (Table 6); the impact of a resection remained significant. In line with the literature in glioblastoma, age was a significant factor upon survival. We further analyzed to what degree the extent of a surgical resection impacted outcome. We found that partial resection did not significantly improve survival, whereas gross-total resection did pose a clear benefit on survival compared to biopsy, which translates into a better outcome for the surgical resection group on the whole (Table 5, Table 6, Fig. 1). Thus, even if 2 out of 3 patients do not receive a gross-total resection due to functional limitations in eloquent areas, the cohort of resected patients still benefits from the mass reduction (Fig. 2).

\section{Discussion}

\section{Treatment of ccGBM—still a matter of debate}

Corpus callosum glioblastomas are rare tumors invading a highly eloquent area of the brain, which results in a difficult treatment decision-making process and argues for a personalized approach. The decisive challenge of treating eloquent tumors lies within the delicate balance between avoiding further neurological impairment and relieving already existing ones. Since a pronounced mass effect of the tumor may rapidly lead to severe neurological deterioration, tumor resection should not be ruled out in general. Meanwhile, an optimal neuro-oncological therapy regime is also required to achieve the maximal survival benefit for the patient. In our study, we sought to address this dilemma and to determine to what extent different treatment strategies affect the neurological and oncological outcomes. Our data showed that the major factor
Table 6 Multivariate Cox regression analyses

Multivariate Cox regression

\begin{tabular}{llll}
\hline & HR & CI 95\% & $p$ value \\
\hline Surgical treatment & & & \\
$\quad$ Biopsy & $*$ & & \\
GTR & 0.35 & $0.13-0.93$ & 0.036 \\
PR & 0.76 & $0.38-1-52$ & 0.431 \\
Adjuvant treatment & & & \\
CT & $*$ & & \\
Palliative & 2.77 & $1.20-6.39$ & 0.017 \\
RCT & 0.81 & $0.34-1.93$ & 0.629 \\
RT & 1.57 & $0.31-7.91$ & 0.586 \\
Anatomy & & & \\
Genu & $*$ & & 0.160 \\
Splenium & 0.41 & $0.12-1.42$ & \\
Sex & & & 0.997 \\
Male & $*$ & & 0.369 \\
Female & 1.00 & $0.53-1.90$ & \\
Epilepsy & 0.70 & $0.33-1.51$ & \\
Age & & & 0.013 \\
Continuous variable & 1.05 & $1.01-1.08$ & \\
KPS & & & \\
Ordinal variable & 0.99 & $0.96-1.02$ & \\
\hline
\end{tabular}

Significant $p$-values are shown italicized

$H R$, hazard ratio; $C I$, confidence interval; GTR, gross-total resection; $P R$, partial resection; KPS, Karnofsky Performance Score

*Reference variable

which drives the decision towards a surgical removal of the tumor was mass effect resulting in a significantly larger tumor volume in the resection cohort, which we tested using a generalized linear model (Supplementary Table 1). We were able to show that this mass effect can be effectively treated with a calculated risk and without postoperative loss of functional performance. This observation shows an important limitation of our retrospective study because large tumors were never

Table 5 Survival analyses

\begin{tabular}{|c|c|c|c|c|c|c|c|}
\hline & \multicolumn{2}{|l|}{ Entire cohort } & \multicolumn{2}{|c|}{ Surgical resection } & \multicolumn{3}{|l|}{ Biopsy } \\
\hline & $N$ & $\%$ & $N$ & $\%$ & $N$ & $\%$ & $p$ \\
\hline Patients & 55 & 100 & 25 & 45.5 & 30 & 54.5 & - \\
\hline Median OS (CI 95\%) & $252(123-513)$ & & $258(153-413)$ & & $220(244-\mathrm{NA})$ & & 0.041 \\
\hline Median PFS & 153 & & 146 & & 154 & & \\
\hline 3-month survival & 42 & 76 & 21 & 84 & 21 & 78 & n.s. \\
\hline 6-month survival & 32 & 58 & 16 & 67 & 16 & 59 & n.s. \\
\hline 12-month survival & 21 & 38 & 12 & 58 & 9 & 33 & 0.032 \\
\hline 24-month survival & 5 & 9 & 3 & 30 & 2 & 7 & 0.047 \\
\hline
\end{tabular}

OS, overall survival; PFS, progression-free survival; n.S., not significant 


\section{a}

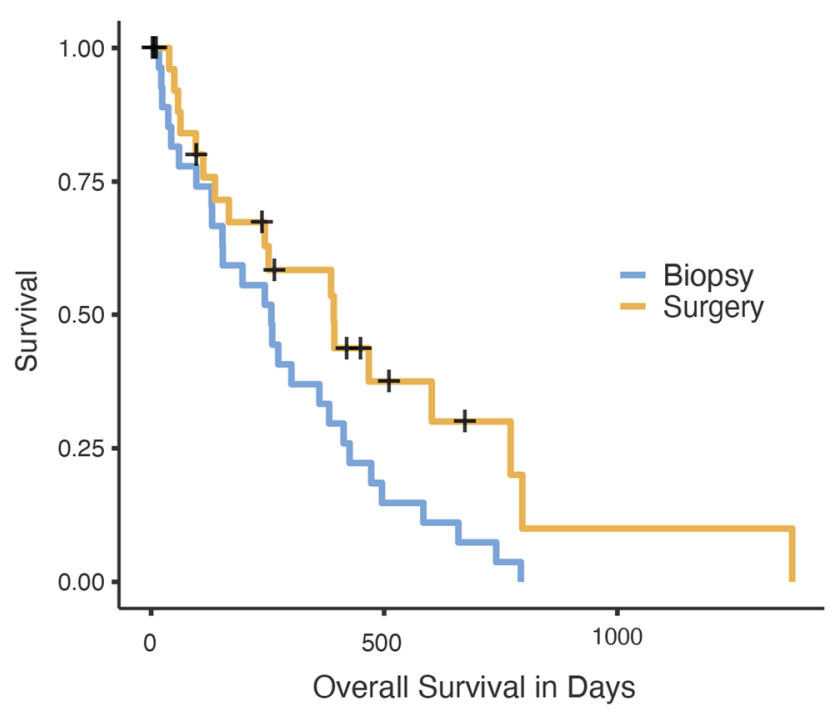

b

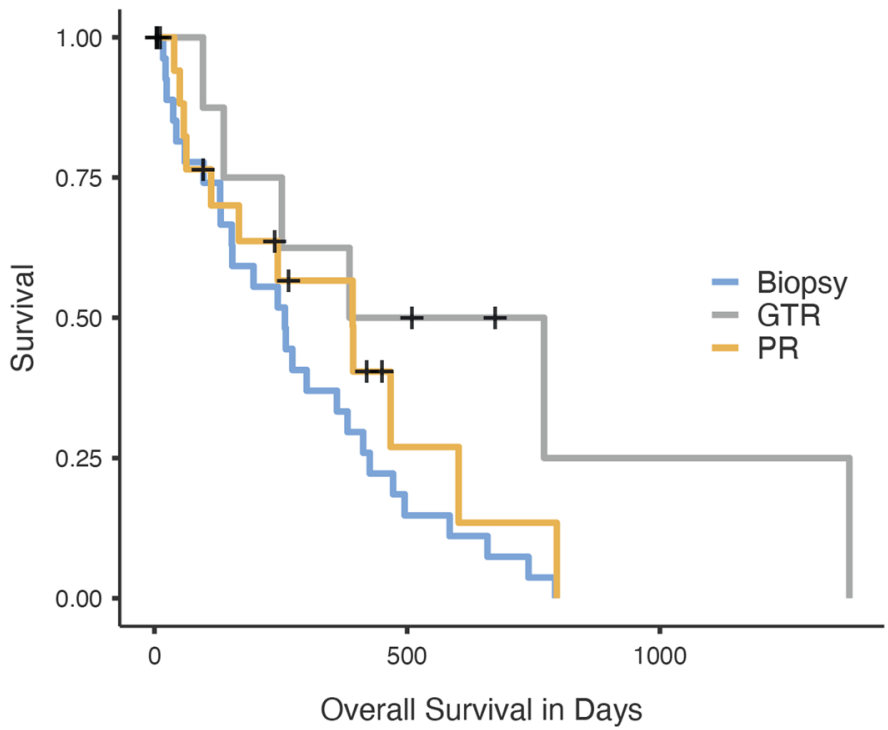

Fig. 1 a Kaplan-Meier analysis of the impact of surgical resection vs. biopsy on survival. b Kaplan-Meier curve for OS stratified by type of surgical approach: gross-total resection, partial resection, and biopsy. GTR, gross-total resection; PR, partial resection

treated by biopsy and adjuvant treatment only due to a high risk of rapidly progressive neurological deterioration and the associated inability to receive adjuvant treatment. The importance of cytoreduction was also reported by Chaichana et al. who was able to show that mass reduction causes favoral neurological outcome and leads to improved functional performance even if the surgical removal comes with higher risk of surgery-associated complications.
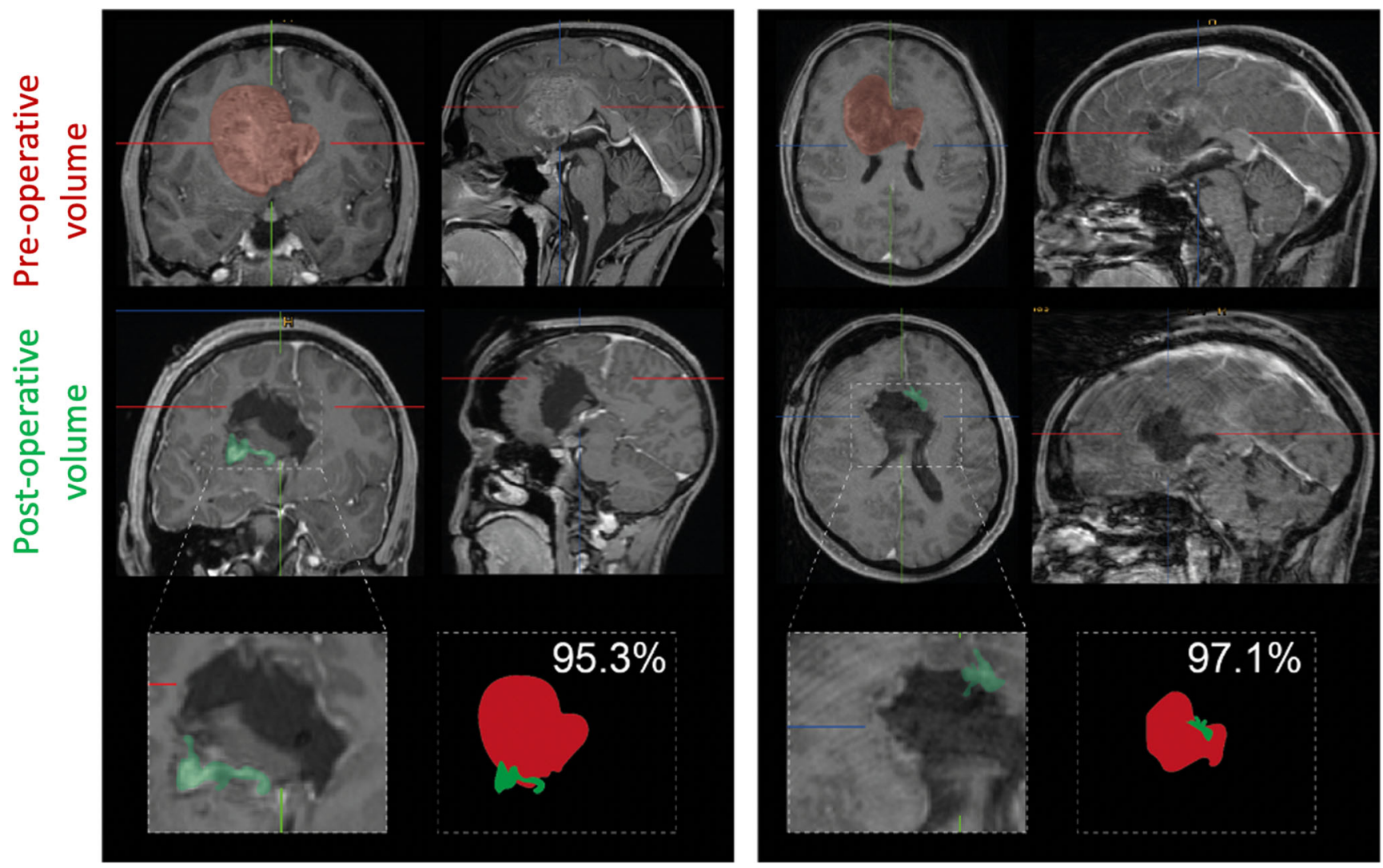

Fig. 2 Two examples of patients with glioblastoma invasion of the corpus callosum who received a gross-total resection; the preoperative (red) as well as the postoperative (residual, green) tumor volumes are illustrated 


\section{Surgical resection improves survival outcome without increasing neurological morbidity}

In line with other authors, we showed a survival benefit for patients who underwent tumor resection [12-14]. However, compared to other published works, the extent of survival benefits is distinctly lower in our cohort. This is due to the improved outcome of the biopsy group. By comparison, already published data indicates a survival of non-resected patients between 1.3 and 3.5 months [12-14, 20]. In contrast, our patients from the biopsy group achieved a median survival of 7.2 months, which is a substantial increase compared to previous reports. This benefit results from a consequent adjuvant treatment of most of the patients, mostly by combined radioand chemotherapy, on the one hand, and the high percentage of lacking adjuvant therapy in comparable studies, on the other hand. Noteworthy, tumor resection improved patients' survival, but did not lead to an increased rate of neurological deficits. Moreover, patients undergoing resection showed better neurological outcome 1 year after treatment compared to biopsied patients. This result could be explained by the fact that the gross-total resections succeeded in reducing tumor volume by more than $95 \%$. This consequently resulted in a meaningful reduction of tumor-associated edema and mass effect-associated symptoms.

\section{Strengths and limitations}

Compared to other studies, our cohort was defined by integrative histopathology including determination of the IDH1/2 mutation by immunohistochemistry (IHC) or nextgeneration sequencing (NGS). From many recent studies, we have learned that mixed histology causes strong bias and misinterpretation of outcome data. Here, we avoid this bias by excluding all patients without clearly defined IDH status. However, a limitation of our study was the reduced number of patients who also had a defined MGMT-Promoter status, due to an uneven integration in the diagnostic work-up at our institution until 2016 (only patients older than 70 years received this assessment routinely).

The cohort of corpus callosum glioma patients is rather small in all previously reported studies and ranges between 29 and 39 cases per institute. Within similar time periods, the number of patients treated at our university was 55. Although our work is based on retrospective analysis, it validates existing findings and supports empirical evidence.

Although ccGBM accounts for only a small percentage of malignant brain tumors, a prospective study which specifically addresses the impact of different treatment modalities on quality of life and neuropsychological and social aspects is necessary to further improve decision-making in these patients.

\section{Conclusion}

Our study aimed to analyze the impact of tumor resection in the largest cohort of IDH wild-type glioma in the corpus callosum (ccGBM) described in the literature thus far. We confirmed that resection of ccGBM is feasible and significantly improves overall survival. In accordance with the common practice of maximum resection in glioblastoma, gross-total resection can be achieved without necessarily leading to aggravation of neurological status. Furthermore, even improvement of functional outcome was possible in gross-totalresected patients compared to biopsied patients at 3-month follow-up. Therefore, surgical resection may be associated with risks, but should be taken into consideration in the management of ccGBM.

Funding Open Access funding enabled and organized by Projekt DEAL.

\section{Compliance with ethical standards}

Conflict of interest The authors declare that they have no conflict of interest.

Ethical approval The local ethics committee of the University of Freiburg approved data evaluation (protocol 472/15). All procedures performed involving human participants were in accordance with the ethical standards of our institution and European standards and with the 1964 Helsinki declaration and its later amendments.

Informed consent Informed consent was obtained from all individual participants included in the study.

Open Access This article is licensed under a Creative Commons Attribution 4.0 International License, which permits use, sharing, adaptation, distribution and reproduction in any medium or format, as long as you give appropriate credit to the original author(s) and the source, provide a link to the Creative Commons licence, and indicate if changes were made. The images or other third party material in this article are included in the article's Creative Commons licence, unless indicated otherwise in a credit line to the material. If material is not included in the article's Creative Commons licence and your intended use is not permitted by statutory regulation or exceeds the permitted use, you will need to obtain permission directly from the copyright holder. To view a copy of this licence, visit http://creativecommons.org/licenses/by/4.0/.

\section{References}

1. Ostrom QT, Gittleman H, Liao P, Vecchione-Koval T, Wolinsky Y, Kruchko C, Barnholtz-Sloan JS (2017) CBTRUS statistical report: primary brain and other central nervous system tumors diagnosed in the United States in 2010-2014. Neuro-Oncology 19(suppl_5):v1-v88

2. VKY H, Reijneveld JC, Enting RH, Bienfait HP, Robe P, Baumert BG, Visser O, Dutch Society for Neuro-Oncology (LWNO) (2014) Changing incidence and improved survival of gliomas. Eur $\mathrm{J}$ Cancer 50(13):2309-2318 
3. Ostrom QT, Gittleman H, Fulop J, Liu M, Blanda R, Kromer C, Wolinsky Y, Kruchko C, Barnholtz-Sloan JS (2015) CBTRUS statistical report: primary brain and central nervous system tumors diagnosed in the United States in 2008-2012. Neuro-Oncology 17(Suppl 4):iv1-iv62

4. Schwartzbaum JA, Fisher JL, Aldape KD, Wrensch M (2006) Epidemiology and molecular pathology of glioma. Nat Clin Pract Neurol 2(9):494-503 quiz $1 \mathrm{p}$ following 516

5. Tamimi AF, Juweid M. (2017) Epidemiology and Outcome of Glioblastoma. In: De Vleeschouwer S, (ed) (Glioblastoma) Codon Publications, Brisbane (AU), pp143-153

6. Stupp R, Mason WP, van den Bent MJ et al (2005) Radiotherapy plus concomitant and adjuvant temozolomide for glioblastoma. $\mathrm{N}$ Engl J Med 352(10):987-996

7. Duffau H, Khalil I, Gatignol P, Denvil D, Capelle L (2004) Surgical removal of corpus callosum infiltrated by low-grade glioma: functional outcome and oncological considerations. J Neurosurg 100(3): 431-437

8. Ho M-L, Moonis G, Ginat DT, Eisenberg RL (2013) Lesions of the corpus callosum. AJR Am J Roentgenol 200(1):W1-16

9. Steltzer KJ, Sauvé KI, Spence AM, Griffin TW, Berger MS (1997) Corpus callosum involvement as a prognostic factor for patients with high-grade astrocytoma. Int J Radiat Oncol Biol Phys 38(1): $27-30$

10. Kallenberg K, Goldmann T, Menke J, Strik H, Bock HC, Stockhammer F, Buhk JH, Frahm J, Dechent P, Knauth M (2013) Glioma infiltration of the corpus callosum: early signs detected by DTI. J Neuro-Oncol 112(2):217-222

11. Krishnan V, Lim TC, Ho FCH, Peh WC (2017) Clinics in diagnostic imaging (175). Corpus callosum glioblastoma multiforme (GBM): butterfly glioma. Singap Med J 58(3):121-125

12. Burks JD, Bonney PA, Conner AK, Glenn CA, Briggs RG, Battiste JD, McCoy T, O'Donoghue DL, Wu DH, Sughrue ME (2017) A method for safely resecting anterior butterfly gliomas: the surgical anatomy of the default mode network and the relevance of its preservation. J Neurosurg 126(6):1795-1811

13. Chaichana KL, Jusue-Torres I, Lemos AM, Gokaslan A, CabreraAldana EE, Ashary A, Olivi A, Quinones-Hinojosa A (2014) The butterfly effect on glioblastoma: is volumetric extent of resection more effective than biopsy for these tumors? J Neuro-Oncol 120(3): 625-634

14. Dayani F, Young JS, Bonte A, Chang EF, Theodosopoulos P, McDermott MW, Berger MS, Aghi MK (2018) Safety and outcomes of resection of butterfly glioblastoma. Neurosurg Focus 44(6): $\mathrm{E} 4$

15. Molinaro AM, Taylor JW, Wiencke JK, Wrensch MR (2019) Genetic and molecular epidemiology of adult diffuse glioma. Nat Rev Neurol 15(7):405-417

16. Delev D, Heiland DH, Franco P, Reinacher P, Mader I, Staszewski O, Lassmann S, Grau S, Schnell O (2019) Surgical management of lower-grade glioma in the spotlight of the 2016 WHO classification system. J Neuro-Oncol 141(1):223-233

17. Heiland DH, Ohle R, Cipriani D et al (2020) Characterization of longitudinal transformation of $\mathrm{T} 2$-hyperintensity in oligodendroglioma. BMC Cancer 20(1):818

18. Wen PY, Macdonald DR, Reardon DA et al (2010) Updated response assessment criteria for high-grade gliomas: response assessment in neuro-oncology working group. J Clin Oncol 28(11):19631972

19. Sanai N, Polley M-Y, McDermott MW, Parsa AT, Berger MS (2011) An extent of resection threshold for newly diagnosed glioblastomas. J Neurosurg 115(1):3-8

20. Opoku-Darko M, Amuah JE, Kelly JJP (2018) Surgical resection of anterior and posterior butterfly glioblastoma. World Neurosurg 110:e612-e620

Publisher's note Springer Nature remains neutral with regard to jurisdictional claims in published maps and institutional affiliations. 